S. marcescens. It did, although only against a less pathogenic strain of the bacterium, possibly because this strain does not produce proteases that degrade the enzyme, whereas the more pathogenic strain does. The authors also assayed $D b l-1$ mutants for their susceptibility to $S$. marcescens infection because $D b l-1$ - a TGF- $\beta$ related gene - regulates some of the genes that were induced in the screen. Dbl-1 mutants were extremely susceptible to $S$. marcescens infection and, surprisingly, also to infection by the Escherichia coli strain OP50, which C. elegans is often cultured on.

So, 30 years after its discovery in Drosophila, these studies show that C. elegans also has an inducible innate-immune response - the components of which are conserved in other organisms - and illustrate the ease with which it can be investigated genetically in worms.

Jane Alfred

Editor, Nature Reviews Genetics

Q) References and links ORIGINAL RESEARCH PAPERS Kim, D. $\mathrm{H}$. et al. A conserved p38 MAP kinase pathway in Caenorhabditis elegans innate immunity. Science 297, 623-626 (2002) | Mallo, G. V. et al. Inducible antibacterial defense system in C. elegans. Curr. Biol. 12, 1209-1214 (2002) FURTHER READING Kimbrell, D. \& Beutler, B. The evolution and genetics of innate immunity. Nature Rev. Genet. 2, 256-267 (2001)

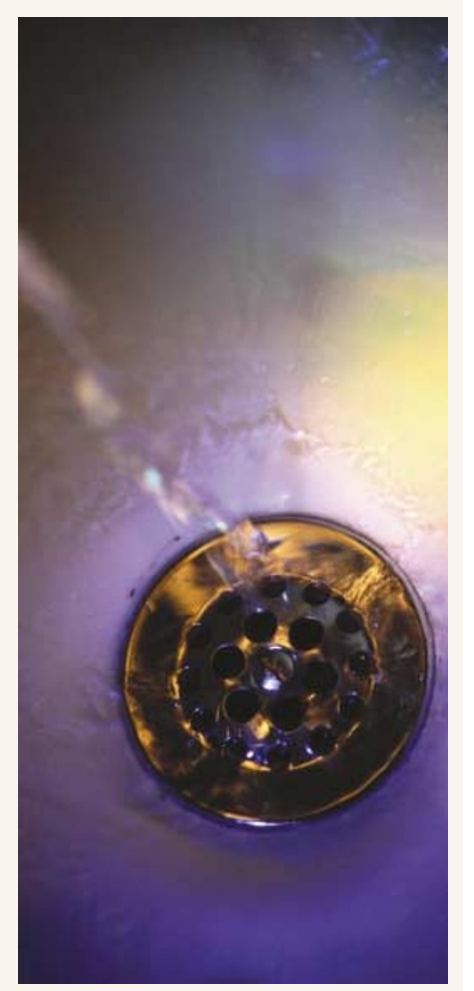

\section{T-CELL SIGNALLING}

\section{The way of CARMA}

This month, our understanding of T-cell receptor (TCR) signalling has been advanced by two studies in Nature Immunology that show that the scaffolding protein CARMA1 links the TCR to nuclear factor- $\mathrm{\kappa} B(\mathrm{NF}-\kappa \mathrm{B})$ activation.

After the recognition of antigen by the TCR, many signalling pathways are triggered simultaneously, which leads to the activation of transcription factors, such as NF- $\kappa \mathrm{B}, \mathrm{NFAT}$ and AP1. NF- $\kappa B$ activation is essential for full T-cell activation, but the upstream signalling pathway is poorly defined. BCL10 - a caspase-recruitment domain (CARD)-containing protein - is known to have a role in TCR-dependent NF- $\kappa B$ activation, as is the novel protein kinase $\mathrm{C} \mathrm{PKC \theta}$, but it is not clear how these components are linked to the TCR. Known binding partners of BCL10 include the CARD-containing subfamily of membraneassociated guanylate kinase (MAGUK) proteins, the CARMA scaffolding proteins.

Gaide et al. looked at the localization of CARMA1 - the only family member that is expressed in lymphocytes - in the Jurkat human T-cell line by immunoprecipitation and confocal microscopy using a polyclonal antibody. They show that CARMA1 is recruited to the TCR after stimulation with anti-CD3 antibody, together with its binding partner BCL10.

How important is the recruitment of CARMA1-BCL10 to the TCR for T-cell activation? Gaide et al. investigated this by generating a mutant CARMA1 protein that cannot bind to BCL10 and that functions as a dominant-negative inhibitor of CARMA1 activity. When Jurkat cells were transfected with the dominant-negative CARMA1 and stimulated with anti-CD3 antibody, NF- $\kappa$ B activation was inhibited markedly and interleukin-2 (IL-2) secretion was completely blocked; however, other TCR signalling pathways, such as the activation of ERK, JNK and PLC $\gamma$, and $\mathrm{Ca}^{2+}$ mobilization, were not affected.

Wang et al. isolated a mutant Jurkat clone in which anti-CD3/anti-CD28 antibody-triggered JNK and ERK signalling was preserved, but $\mathrm{NF}-\kappa \mathrm{B}$ activation was disrupted. This defect was shown to be downstream of PKC $\theta$ and upstream of IKK (inhibitor of NF- $\mathrm{KB}$ kinase) and to prevent IL-2 expression. The mutant cells are defective in CARMA1 expression, and reconstitution with CARMA1 rescued the defect in both NF- $\mathrm{KB}$ activation and IL-2 expression. This result provides genetic evidence that CARMA1 is an essential signalling component in the TCR signalling pathway. The authors also show that CARMA1 is essential for optimal recruitment of

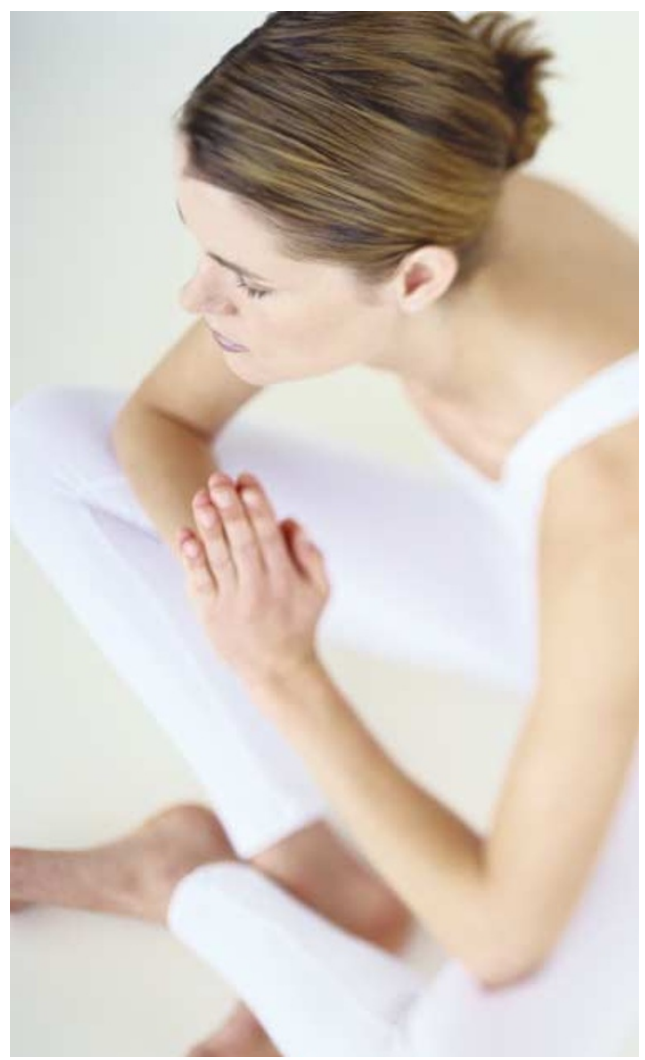

BCL10 to the membrane and that it enhances the phosphorylation of BCL10 by PKCO.

It has been proposed that early $\mathrm{T}$-cell signalling events are initiated in membrane lipid microdomains known as rafts; so, are CARMA1 and BCL10 recruited to the rafts? Using confocal microscopy, Gaide et al. show that CARMA1 is enriched in rafts after cross-linking of rafts. A biochemical analysis of anti-CD3-stimulated Jurkat $T$ cells, using detergents to isolate rafts, showed that CARMA1 is present constitutively in the raft fraction and that its concentration is increased after TCR stimulation. BCL10, which is excluded from rafts in resting cells, also translocates to rafts after stimulation. It is not clear how CARMA1 is targeted to rafts, but the authors' preliminary results indicate that CARMA1 might bind to a raft protein component, possibly through one of its protein-protein interaction domains.

Together, these papers indicate that CARMA1 has an essential and specific role in TCR-mediated NF- $\mathrm{KB}$ activation; it recruits BCL10 to the TCR in rafts and allows the activation of BCL10 - either directly or indirectly - by PKCO.

Jennifer Bell

(1) References and links ORIGINAL RESEARCH PAPERS Gaide, O. et al. CARMA1 is a critical lipid raft-associated regulator of TCR-induced NF-KB activation. Nature Immunol. August 52002 (DOI 10.1038/ni830) | Wang, D. et al. A requirement for CARMA1 in TCR-induced NF-KB activation. Nature Immunol. August 52002 (DOI 10.1038/ni824)

WEB SITES

Xin Lin's lab: http://www.smbs.buffalo.edu/wcmpi/faculty/xin_lin.htm Margot Thome's lab:

http://www.unil.ch/ib/groups/tschopp/thome/thome.html 